\title{
La Dimensión Estratégica de la Política de Defensa: apuntes para su conceptualización desde el caso argentino*
}

The Strategic Dimension of Defense Policy: an approach for the conceptualization from the Argentine case

\author{
EZEQUIEL MAGNANI** \\ Universidad Torcuato Di Tella, Argentina \\ Consejo Nacional de Investigaciones Científicas y Técnicas, Argentina \\ ezequielmagnani11@gmail.com
}

https://doi.org/10.46468/rsaap.15.1.A4

\begin{abstract}
Resumen: El presente artículo indaga la vertiente estratégica de los estudios sobre defensa nacional y destaca a la misma como una línea de investigación que ha sido escasamente abordada por la literatura. Luego de posicionar dicha vertiente en el campo, se establecen puentes conceptuales entre los términos "defensa" y "estrategia" con el objetivo de tener una mejor aproximación teórica y analítica a lo que denominaremos la "Dimensión Estratégica de la Política de Defensa" (DEPD). Asimismo, se identifica que la misma está compuesta por cuatro elementos que, si bien están estrechamente relacionados, pueden ser analizados por separado. En este sentido, se utiliza el caso argentino para abordar, mediante el análisis de documentos oficiales, leyes vigentes y entrevistas en profundidad a actores clave, a cada componente de la DEPD e ilustrar tanto la vinculación entre ellos como la relación entre el concepto y el caso empírico utilizado.
\end{abstract}

Palabras clave: Defensa nacional - Estrategia - Seguridad internacional - Relaciones Internacionales - Argentina

\begin{abstract}
This article inquires about the strategic subfield of the studies of national defense and highlights it as a strand that has been scarcely addressed by the literature. After positioning the subfield in the studies on national defense, bridges are established between the concepts 'defense' and 'strategy' with the aim of having a better theoretical and analytical approach to what we will call 'Strategic Dimension of the Defense Policy' (SDDP), indicating that it is made up of four elements that, although closely related to each other, can be analyzed separately. In this sense, the Argentine case is used to address, through the analysis of official documents, current laws and conducting in-depth interviews with key actors; each component of the DEPD and illustrate both the link between them and the relationship between the concept and the empirical case used.
\end{abstract}

Key words: National Defense - Strategy - International Security - International Relations - Argentina

* Artículo recibido el 20 de julio de 2020 y aceptado para su publicación el 12 de marzo de 2021.

** El autor agradece el aporte de evaluaciones anónimas a una versión previa de este artículo. 


\section{Introducción}

El presente trabajo tiene como objetivo indagar y profundizar sobre la dimensión estratégica de la política de defensa, considerando que es una línea de investigación que ha recibido poca atención por parte de la literatura. A pesar de que ésta es una vertiente poco explorada en la actualidad, los estudios sobre defensa nacional en la Argentina abordan cuestiones que son de vital importancia para reflexionar y analizar las múltiples dimensiones de la política de defensa del país. En este sentido, es destacable la proliferación en las últimas dos décadas de estudios vinculados a las relaciones cívico-militares y a la conducción política de la defensa nacional (López, 1988), a los procesos de planeamiento, formulación e implementación de la política de defensa (Eissa, Gastaldi, Canto y Justribó, 2013) y a los llamados "nuevos temas" (Ramos, 2015). No obstante, pese a la multiplicidad de líneas de investigación, la mayoría de ellos no profundiza en las cuestiones vinculadas al carácter estratégico de la política de defensa. A su vez, los autores que tratan temas vinculados a las cuestiones estratégicas suelen hacerlo de forma indirecta, relacionando a esta dimensión estratégica con cuestiones relativas al planeamiento o a la normativa que rige el empleo del instrumento militar (Scheetz y Cáceres, 1995). De esta manera, el presente trabajo parte de la premisa de que la dimensión estratégica de la política de defensa, al abarcar un contenido distinto al de las otras dimensiones, puede abordarse de forma directa; conceptualizando, definiendo, explicando y relacionando sus atributos particulares al mismo tiempo que se diferencia y complementa a los abordajes más tradicionales.

En esta línea, antes de comenzar a conceptualizar lo que en este trabajo llamaremos la "Dimensión Estratégica de la Política de Defensa" (DEPD), será necesario establecer la forma en la que este artículo se inserta y complementa a la literatura existente. Esta tarea reviste especial importancia ya que el desarrollo conceptual de la DEPD requiere reconocer y marcar la profunda vinculación de ésta con algunas consideraciones fundamentales de los estudios precedentes.

Asimismo, indagaremos sobre el contenido tanto del concepto de "defensa nacional" como el de "estrategia", con el objetivo de identificar puentes entre ambos, algo que es necesario para establecer un piso teórico sólido a partir del cual desarrollar la DEPD. De tal forma, las varias conceptualizaciones de "estrategia" provenientes de autores más tradicionales y de los autores contemporáneos, vistas a la luz de los objetivos y el significado de la "defensa nacional", nos permitirá dar cuenta de cómo el presente artículo amplía el campo de estudio al proponer nuevas líneas de investigación vinculadas a la dimensión estratégica. 
Luego de haber desarrollado esta vinculación conceptual y la forma en la cual el presente artículo se posiciona y complementa a los estudios académicos existentes, procederemos a desarrollar la DEPD. La aproximación a la dimensión estratégica se realizará a partir de los cuatro elementos que integran la misma y que refieren a cuatro preguntas principales que deben ser contestadas por un Estado, a saber: (1) ¿Qué objeto/s referente/s se busca/n defender? (2) ¿Cómo se piensa defenderlo/s? (3) ¿De quién hay que defenderlo/s? (4) ¿En qué escenario hay que defenderlo/s? Es a partir de estas interrogantes que podemos comenzar a conceptualizar los cuatro componentes de la DEPD, ya que cada uno de ellos contribuye a la comprensión general de esta faceta de la defensa nacional. En este sentido, la segmentación realizada en función de las interrogantes tiene el objetivo de facilitar su comprensión en términos analíticos, teniendo siempre en consideración que estos componentes nunca se dan por separado, sino que están fuertemente entrelazados y solo la consideración y el análisis de los cuatro en simultáneo puede brindar una aproximación certera a la DEPD de un determinado Estado.

La definición de la política de defensa en términos generales como "el conjunto de acciones que adopta un Estado para garantizar su supervivencia frente a riesgos y amenazas" (Battaglino, 2010: 242) también pone en valor esta vertiente estratégica, ya que solo ésta aborda de forma directa los temas relativos a la protección del Estado, a los medios necesarios para asegurarla, a la percepción de amenazas y al reconocimiento del escenario regional e internacional. De esta manera, al indagar tanto respecto a los factores objetivos a proteger dentro del territorio nacional como a las precepciones de amenaza y del marco regional e internacional, el presente trabajo destaca la profunda vinculación entre la dimensión estratégica de la política de defensa y el contexto mundial en el cual está inserto un determinado Estado. Esta relación es estrecha en la medida que, si bien los objetos a defender y los medios para hacerlo son siempre pensados en clave nacional, los mismos únicamente adquieren sentido a partir de la consideración del plano internacional. En términos metodológicos, el artículo adopta un enfoque cualitativo en tanto se utiliza el estudio de caso argentino a partir del análisis de documentos oficiales, leyes vigentes y la realización de entrevistas en profundidad a informantes clave con el objetivo de ilustrar empíricamente la DEPD.

El desarrollo del artículo continúa de la siguiente manera. En la próxima sección explicaremos la forma en la que el desarrollo de la DEPD parte de fundamentos establecidos por gran parte de la literatura precedente. En la segunda sección estableceremos los puentes entre el concepto de "defensa" y el de "estrategia" con el objetivo de ampliar el espectro de análisis de 
estos estudios y, de esta forma, tener un piso teórico sólido a partir del cual desarrollar la DEPD. En la tercera sección desarrollaremos la DEPD, introduciendo sus cuatro componentes y explicando la manera en la que los mismos se relacionan. En la última sección, estableceremos las conclusiones haciendo especial énfasis en los aportes y las ventajas que este nuevo concepto brinda a los estudios sobre defensa nacional.

\section{Lugar en la literatura}

A partir de 2003 comenzó a tener lugar una creciente revalorización de los estudios sobre la defensa que llevó a una progresiva multiplicación de las publicaciones académicas caracterizada por el desarrollo de tres tipos de vertientes dentro del campo (Ramos, 2015). En primer lugar, aquellos que retoman y profundizan los estudios tradicionales vinculados a las relaciones cívico-militares (Battaglino, 2010; Derghougassian, 2012). En segundo lugar, los estudios que abordan la cuestión relativa a la formulación e implementación de la política de defensa, en donde se vincula al planeamiento estratégico con cuestiones ligadas a la política militar, a factores institucionales, presupuestarios y estratégicos (Eissa, Gastaldi, Canto y Justribó, 2013; Eissa, 2013a). En tercer lugar, aquellos ya mencionados y que pueden catalogarse bajo el rótulo de “nuevos temas” (Ramos, 2015).

Ante este escenario, el presente artículo se inserta en la literatura existente y surgida a partir de 2003 al incorporar como premisas argumentos centrales establecidos en ésta y, a su vez, complementa a los estudios sobre defensa en la medida que indaga conceptualmente sobre un tema virtualmente inexplorado hasta el momento.

La conceptualización de la DEPD asume como premisas cuatro argumentos ya presentes en la literatura sobre defensa nacional. El primero retoma los estudios vinculados a las relaciones cívico-militares desarrollados por varios expertos sobre defensa (Saín, 2010; Anzelini, Poczynok y Zacarías, 2017; Pion-Berlin y Martinez, 2019) con el objetivo de hacer hincapié en el hecho de que cualquier conceptualización de la DEPD debe partir de la idea de que las definiciones, lineamientos y directivas estratégicas siempre tienen como premisa la conducción política del sistema de defensa nacional. En tal sentido, la DEPD en su conceptualización original ve al instrumento militar como un componente esencial para la realización de sus objetivos estratégicos, pero no como la piedra angular a partir de la cual éstos se desprenden.

En esta misma línea, el segundo argumento que asume la DEPD y sobre el cual se desarrolla el concepto es la vigencia y consolidación de lo que los 
estudios de defensa denominan "consenso básico". El mismo se enmarca en tres leyes esenciales -la Ley 23.554 de Defensa Nacional (1988); la Ley 24.059 de Seguridad Interior (1992); y la Ley 25.520 de Inteligencia Nacional (2001)- que rigen el campo de acción del instrumento militar y que, como mencionan Canto, Eissa y Gastaldi (2015), deben ser leídas de manera conjunta con el objetivo de identificar un constructo sistémico articulado en tres principios: "(1) La supresión de las hipótesis de conflicto con los países vecinos que requieran la utilización de las Fuerzas Armadas; (2) la separación orgánica y funcional entre defensa nacional y seguridad interior; y (3) el gobierno civil de la política de defensa" (Canto, Eissa y Gastaldi, 2015: 6). De esta manera, la DEPD toma a los mismos como los lineamientos principales a partir de los cuales se enmarca y define sus componentes. Esta cuestión es de relevancia en la medida que tiene un doble efecto. Por un lado, limita el espectro estratégico del concepto al eliminar la posibilidad de utilización de la defensa nacional para asuntos vinculados a la seguridad interior como pueden ser el crimen transnacional organizado o la lucha contra el terrorismo. Por otro lado, complejiza la dimensión estratégica ya que ésta deja de estar guiada solamente por una hipótesis de conflicto en donde el cálculo estratégico está vinculado a cuántos medios materiales son necesarios para hacerle frente a otro Estado.

El tercer argumento está vinculado con la relevancia del planeamiento estratégico para la dimensión estratégica de la política de defensa. En relación con esto, a partir del Decreto 1729/2007 que aprueba el Ciclo de Planeamiento de la Defensa Nacional (CPDN) -concebido como la instancia en donde se "organiza y encuadra el proceso de definición estratégica" (Decreto 1729/2007, art. 2) - comenzaron a aparecer estudios vinculados a la conceptualización, definición e importancia del planeamiento estratégico militar, haciendo hincapié en el planeamiento por capacidades en reemplazo del modelo basado en hipótesis de conflicto (Decreto 1691/2006). Estos estudios destacan la ventaja de este cambio en la medida que "el diseño de fuerzas se acomoda al desarrollo de los medios militares de probable empleo, en base a la defensa de los intereses vitales identificados por el Nivel Estratégico Nacional, en el marco de una actitud estratégica defensiva" (Anzelini y Poczynok, 2014: 156).

Los estudios vinculados al planeamiento y al posicionamiento estratégico que define un Estado son de utilidad en tanto, si bien buscan abordar las cuestiones estratégicas desde una perspectiva normativa y procedimental, se aproximan a la conceptualización teórica de la DEPD. Esto es así ya que a lo largo de todo el proceso de planeamiento estratégico se requiere la identificación de tres de los componentes esenciales vinculados a la DEPD que 
propondremos en el presente artículo. Estos son: (1) la identificación de los intereses a defender -intereses vitales definidos por el Nivel Estratégico Nacional según Anzelini y Poczynok (2014)-, (2) las capacidades necesarias para defender dichos intereses -que se establecen a lo largo de todo el CPDN establecido por el decreto 1729/2007-y (3) los potenciales escenarios vinculados a las amenazas estatales que pueden requerir el empleo del instrumento militar -que hace estricta referencia al planeamiento por capacidades. En este sentido, la DEPD retoma cuestiones identificadas por los estudios relativos al planeamiento estratégico y los complementa al alejarse del abordaje normativo y procedimental para buscar profundizar tanto la conceptualización teórica de estos atributos identificados como la relación entre ellos.

Ahora bien, con respecto al último argumento sostenido por la literatura más reciente sobre defensa y a partir de los cuales desarrollamos la DEPD, destacamos aquellos estudios que indagan acerca de los "déficits de atención" y que reflexionan respecto del motivo por el cual amplios sectores políticos en América Latina ignoran la política de defensa (Pion Berlin y Trinkunas, 2007; Battaglino, 2015). Dentro de esta nueva vertiente, destacamos el trabajo de Battaglino (2015) en la medida que contribuye a demarcar el campo disciplinar a partir del establecimiento de tres niveles de análisis: (1) el de la definición conceptual de la defensa nacional, (2) el nivel de los medios (materiales y no materiales) y (3) el de los fundamentos de la defensa, siendo esta última "la más decisiva, pues define los recursos materiales y simbólicos que un estado le destinará” (Battaglino, 2015: 200). Tanto el nivel de la definición conceptual como el de los medios ya han sido abordados. El primer nivel de análisis fue considerando a partir de aquellos intereses vitales que un Estado busca defender, siendo en el caso argentino la integridad territorial y la autodeterminación del pueblo los objetos referentes a defender y únicamente las amenazas estatales externas aquellas que pueden poner en riesgo los mismos (Ley 23.554; Decreto 727/2006; Decreto $571 / 2020)$. El segundo nivel es abordado en el caso argentino a partir del CPDN, en donde se definen los medios necesarios en función de los escenarios de probable empleo del instrumento militar. En lo que respecta al tercer nivel relativo a los fundamentos, su conceptualización como "las condiciones políticas que incentivan, o no, el interés de los políticos y la sociedad en la defensa nacional. (...) los fundamentos permiten dar cuenta de las variaciones en los medios, es decir, son su variable independiente" (Battaglino, 2015: 207) hace que sea un insumo fundamental para la conceptualización de la DEPD. Esto es así no solo porque el factor vinculado a los medios -cómo se piensa defender los objetos referentes- es uno de los 
cuatro componentes de la DEPD, sino porque sostenemos que los fundamentos permiten dar cuenta de las variaciones en todos sus componentes en la medida que éstos están siempre sujetos a definiciones de carácter político.

De esta forma, la DEPD se inserta en la literatura sobre defensa nacional argentina al desarrollarse conceptualmente a partir de cuatro argumentos clave sostenidos por los estudios existentes. Estos son: (1) la subordinación política del instrumento militar, (2) la plena vigencia del "consenso básico", (3) la relevancia del planeamiento estratégico y sus contribuciones al desarrollo de la DEPD al identificar e incluir en el CPDN parte de sus componentes y, por último, (4) la relevancia de los fundamentos de la defensa, ya que los componentes de la DEPD requieren siempre una definición de carácter político.

\section{La defensa nacional y su vinculación con la estrategia}

Establecer puentes teóricos que vinculen al concepto de 'defensa nacional' con el de 'estrategia' es una empresa compleja debido al hecho que aluden a cuestiones que $a$ priori son distintas. Mientras la primera hace referencia a un objetivo a cumplir por el aparato estatal, la segunda hace alusión a una multiplicidad de cuestiones vinculadas con la mejor forma de lograr un determinado objetivo que no necesariamente está ligado a la instancia estatal. En otras palabras, la defensa nacional no puede analizarse por fuera del Estado, mientras que la estrategia puede aplicarse a muchas dimensiones que exceden dicho plano. Sin embargo, a la hora de pensar en una política de defensa, es muy difícil no vincular a la misma con el término 'estrategia', ya que al momento de pensar cómo llevar adelante la misma, es inevitable que surjan interrogantes vinculados a la mejor forma de hacerlo (Anzelini y Poczynok, 2014).

Para precisar conceptualmente ambos términos y su relación, es de utilidad volver a los escritos de Karl Von Clausewitz. Si bien es un autor clásico frecuentemente citado para los estudios vinculados a las relaciones cívicomilitares $^{1}$, sus reflexiones sobre la defensa y la estrategia, presentes a lo largo de toda su obra, nos brindan herramientas fundamentales para definir dichos conceptos y establecer una base sólida para luego poder desarrollar las conceptualizaciones más contemporáneas de los mismos.

Estos estudios hacen frecuente a la alusión de que "la guerra es la mera continuación de la política por otros medios" (Clausewitz, 2015: 48). 
En lo que respecta a la defensa, Clausewitz reflexiona:

¿Cuál es el objetivo de la defensa? Detener un golpe. ¿Cuál es, entonces, su signo característico? La espera de ese golpe. Este es el signo que hace de cualquier acto un acto defensivo, y solo mediante este signo la defensa puede distinguirse en la guerra del ataque (Clausewitz, 2015: 247).

En esta misma línea, al continuar con su argumentación, el autor precisa la cuestión vinculada a los objetivos, mencionando que el fin último de la defensa es el de la preservación (Clausewitz, 2015: 248). Asimismo, a la hora de abordar cómo debe ser "la espera" de ese golpe, establece que "no debe ser mera subsistencia pasiva, y que la acción ligada a ella en la destrucción de las fuerzas enemigas empeñadas en la lucha puede ser el propósito, al igual que cualquier otro" (Clausewitz, 2015: 63).

A partir de estas referencias, podemos identificar tres cuestiones principales que están presentes en su conceptualización sobre la defensa y que aparecen como certezas. En primer lugar, que el concepto responde a algo que debe ser preservado; en segundo lugar, que ese objeto a ser preservado está sujeto a una acción que debe realizarse de determinada manera; y, en tercer lugar, que la mencionada acción corresponde a detener una conducta hostil proveniente de un actor que amenaza y pone en riesgo el objeto que se busca preservar. Además de estos factores, Clausewitz también hace alusión al contexto en el cual tiene lugar la defensa al abordar la relación existente entre el terreno y la guerra, argumentando que es "imposible concebir que una acción bélica de parte de nuestro ejército organizado se produzca de otro modo que en un espacio definido (...) $[\mathrm{y}]^{2}$ como regla uno de los dos combatientes (el defensor) conoce mucho más acerca del lugar" (Clausewitz, 2015: 78). En consecuencia, es posible identificar una cuarta cuestión relativa a la conceptualización del término 'defensa', y es que la misma no se desarrolla en un vacío, sino que tiene lugar en un escenario determinado.

En consideración de estos cuatro componentes identificados en el pensamiento de Clausewitz, es destacable que los mismos siguen estando vigentes en los trabajos más actuales sobre la defensa nacional. Como fue mencionado, Battaglino (2015) contribuye a definir el campo disciplinar al identificar tres niveles de análisis: el correspondiente a la definición conceptual, el de los medios y el de sus fundamentos. Si bien es evidente que la cuestión de los fundamentos no puede identificarse con las reflexiones de

Añadido por el autor. 
Clausewitz, los puntos relativos a la definición conceptual y a la de los medios están vinculadas, por un lado, al objeto que debe ser preservado y, por otro lado, a la acción que debe realizarse para defenderlo. A su vez, Battaglino define a la política de defensa como "el conjunto de acciones que adopta un Estado para garantizar su supervivencia frente a riesgos y amenazas" (Battaglino, 2010: 242), en donde podemos destacar en la literatura contemporánea ya no solo las cuestiones vinculadas al objeto a preservar y a las acciones que debemos tomar para hacerlo, sino también el hecho de que esas dos cosas deben hacerse teniendo en consideración aquellos actores externos y hostiles que pueden actuar como amenaza.

En esta misma línea de investigación, Eissa (2013b) retoma la definición de Battaglino, ampliando el campo de estudio relativo a la defensa nacional al establecer que la misma contiene tres dimensiones. La primera es la ya mencionada política militar correspondiente a la subordinación política del instrumento militar (Battaglino, 2012a). La segunda es la estratégica, en donde el autor parafrasea a Saín y define a dicha dimensión como

las acciones, actitudes y medidas institucionales de carácter estratégico, decididas e implementadas por el gobierno nacional y destinadas a prevenir o enfrentar distintos tipos de situaciones de riesgo, conflictos o amenazas, potenciales o efectivas, que provengan de agresiones estatales de origen externo y que hagan peligrar la integridad territorial y la capacidad de autodeterminación del Estado y que requieran el empleo de las Fuerzas Armadas, en forma disuasiva o efectiva. (Saín 2003b: 234 en Eissa, 2013a: 174-175).

Esta definición es complementada por Eissa (2013a), a partir de un abordaje normativo de las leyes y decretos que delimitan el accionar del instrumento militar con el objetivo de identificar, por un lado, qué tipo de acciones deben ser implementadas y contra qué potenciales amenazas; $y$, por otro lado, cómo estas acciones contribuyeron a la política exterior Argentina. Si bien encontramos muy valiosa la definición de la dimensión estratégica, el presente artículo tiene como objetivo complementarla en la medida que ésta no menciona las conexiones teóricas y prácticas existentes entre el concepto de 'defensa' y el de 'estrategia'. En este sentido, al asumir de forma asertiva que hay acciones, actitudes y medidas que por sí mismas son de carácter estratégico, nos brinda la posibilidad, en este trabajo, no solo de establecer la mencionada relación conceptual, sino también de poder definir analíticamente los componentes de la dimensión estratégica, estableciendo las relaciones entre ellos y profundizando su abordaje teórico. 
Ahora bien, con respecto a la dimensión internacional de la política de defensa y retomando los elementos identificados a partir de las reflexiones de Clausewitz, el autor afirma que la misma "se constituye en punto de contacto entre la política de defensa (en términos generales) y la política exterior" (Eissa, 2013a: 175). En tal sentido, si bien el objetivo de su artículo no es profundizar en la identificación y reconocimiento de los componentes analíticos que distinguen a esta dimensión internacional, su argumentación asociada al hecho de que la política de defensa tiene la capacidad de contribuir a la política exterior nos permite pensar que el escenario regional e internacional bajo el cual tiene lugar la defensa nacional también tiene un impacto en la misma.

En función de lo dicho, es posible afirmar que la conceptualización de la "defensa nacional" parte de cuatro certezas que son intrínsecas al propio concepto. La primera es que hay un objeto referente que debe ser defendido; la segunda está vinculada con el hecho de que esta defensa debe realizarse de determinada manera y a partir de medios concretos; la tercera refiere a que la defensa debe ser realizada contra otro actor que amenaza al objeto que se quiere preservar; y, por último, la cuarta está relacionada al reconocimiento que esta acción se da siempre en un determinado escenario. Dicho de otro modo, el concepto en cuestión se constituye a partir de cuatro certezas que guían a todos los Estados a la hora de definir su política de defensa. Esto es así ya que cualquiera de los actores estatales que busca diseñar e implementar una, lo hace debido a que tiene la intención de establecer la mejor forma de preservar un objeto que es o puede ser amenazado por un actor hostil en un determinado escenario regional o internacional.

Si el concepto de 'defensa' se constituye a partir de las certezas, es posible adentrarnos en el de 'estrategia' sosteniendo que puede ser abordado desde la incógnita. En este sentido, podemos establecer el origen de dicho término a partir de los escritos de Tucídides en su famosa obra La Historia de la Guerra del Peloponeso en el siglo IV a.C, en donde relata los movimientos estratégicos en la guerra, tanto de la coalición liderada por los atenienses como de la conducida por los espartanos. También son destacables las reflexiones de Sun Tzu en sus escritos en El arte de la Guerra, a partir de los que establece los cinco fundamentos de la estrategia y la importancia que los mismos revisten para la supervivencia de un país (Sun Tzu, 2014: 17). En esta misma línea, entre los autores más contemporáneos que mantienen su base argumentativa en la importancia de la estrategia en el escenario de la guerra, podemos encontrar a Karl Von Clausewitz con su obra De la Guerra, a Alfred Mahan a partir de sus reflexiones sobre la estrategia y el poder naval y, por último, a Sir Basil H. Liddell Hart con su libro Estrategia. La aproximación indirecta. 
Si bien el concepto de 'estrategia' es la piedra angular de todos los escritos mencionados, es Clausewitz quien lo sistematiza de la mejor manera, incluyéndolo en un constructo teórico que busca abarcar las varias facetas de la guerra, entre las que se encuentran la defensa, el ataque, la táctica, el genio militar y los objetivos políticos. En su obra, el autor se refiere a la estrategia como

el uso del encuentro para alcanzar el objetivo de la guerra. Por lo tanto, debe dar un propósito a toda la acción militar, propósito que debe estar de acuerdo con el objetivo de la guerra. En otras palabras, la estrategia traza el plan de guerra y, para el propósito mencionado, añade las series de actos que conducirán a ese propósito; o sea, hace los planes para las campañas separadas y prepara los encuentros que serán librados en cada una de ellas (Clausewitz, 2015: 171).

Dos elementos pueden identificarse en esta definición que son relevantes para el objetivo de este artículo. En primer lugar, que la estrategia siempre responde a la consecución de un objetivo político que le da sentido, es decir, que la orienta y permite medir su éxito. En segundo lugar, que la misma responde a un proceso creativo en donde la única certidumbre es el objetivo a conseguir. En otras palabras, la estrategia se desarrolla sobre la incógnita relativa a cuál es el mejor medio para llegar al fin político establecido. En palabras de Clausewitz: "en la estrategia, donde todo se mueve mucho más lentamente, hay mucho más lugar para nuestras propias dudas y la de los otros, para las objeciones y las protestas, y, en consecuencia, también para los remordimientos inoportunos" (Clausewitz, 2015: 173).

A partir de los autores clásicos mencionados, Bueno (2018) -al demarcar el campo profesional de los estudios estratégicos- establece que, luego de la aparición de las armas nucleares, el concepto de 'estrategia' se revitalizó en los estudios académicos y comenzó a ser vinculado con el nivel político de toma de decisión. En este nuevo escenario, la literatura relativa a los estudios estratégicos empezó a hacer referencia al impacto político del poder militar (Garnett, 1987; Gray, 1999; Kane y Lonsdale, 2012; Vennesson, 2017) y al uso de la estrategia en tiempos de paz (Earle, 1943, en Heuser, 2010). En consecuencia, una aproximación actual al concepto es aquella que refiere al mismo como "el logro de los fines políticos deseados, a través de la elección de los modos estratégicos adecuados, empleando en gran medida los medios militares disponibles o accesibles" (Gray, 2015: 10).

De esta manera, la literatura relativa a los estudios estratégicos mantiene los dos elementos identificados a partir de las reflexiones de Clausewitz. 
Por un lado, que ésta siempre responde a un fin político y, por otro lado, que la misma parte de un interrogante en la medida que quien la lleva adelante debe preguntarse por cuál es el mejor medio para alcanzar el objetivo establecido. En este sentido, es posible argumentar que mientras que el concepto de 'defensa nacional' está vinculado con la certeza, el concepto de 'estrategia' tiene su origen en la incertidumbre propia a la relación medio-fin. Dicho de otro modo, el primero parte del conocimiento de que hay un objeto referente que debe ser defendido, a diferencia del segundo que se desarrolla a partir de la incertidumbre de no tener seguridad sobre cuáles son las mejores acciones a realizar para lograr la meta fijada. En consecuencia, la estrategia siempre parte del cuestionamiento y la duda.

Ahora bien, ¿cuál es la relación entre ambos términos que nos permite combinarlos y argumentar que la política de defensa tiene una dimensión estratégica? Podemos establecer que los mismos se relacionan armónicamente en la medida en que el segundo complementa al primero, completándolo teóricamente y dándole una dimensión que permite ampliar su contenido. Los puentes entre ambos conceptos son tan estrechos que es casi imposible pensar a la defensa nacional por fuera de su relación con la estrategia, ya que no solo es suficiente con tener las certezas sobre que hay que defender un objeto, de alguna forma, de alguien y en un contexto; sino que también hay que tener en claro cuál es ese objeto referente que se quiere preservar, cómo se debe hacerlo, de quién se debe defenderlo y en qué contexto. En tal sentido, la 'estrategia' viene a problematizar las certezas de la 'defensa nacional', colocándose sobre ellas y ampliando su alcance de análisis en tanto sus interrogantes le dan utilidad práctica a la defensa nacional al permitir establecer resoluciones estratégicas de carácter político vinculadas a lo que debe ser defendido, cómo, de quién y en qué escenario.

De esta forma, la DEPD surge a partir de la combinación de las certezas propias del concepto de 'defensa nacional' con la problematización propia de la 'estrategia' vinculada a las interrogantes ligadas a la mejor forma de llegar a un determinado fin. Esta relación permite llegar a cuatro tipos de resoluciones estratégicas que, a su vez, sostenemos que son las que componen la DEPD en la medida que las mismas son la síntesis que surge de la relación entre ambos conceptos. Estas resoluciones estratégicas son: en primer lugar, la definición del/de los objeto/s referente/s a defender; en segundo lugar, el establecimiento de las mejores formas de defender el/los objeto/ s referente/s; en tercer lugar, la identificación de los actores que son potenciales amenazas para el objeto referente; y, en cuarto lugar, el reconocimiento del escenario bajo el cual será posible defender el objeto referente. 
Ezequiel Magnani

\section{TABLA 1}

Dimensión Estratégica de la Política de Defensa

\begin{tabular}{|l|l|l|}
\hline \multicolumn{2}{|c|}{ Dimensión Estratégica de la Política de Defensa } \\
\hline Certezas de la defensa & $\begin{array}{l}\text { Interrogantes de la } \\
\text { estrategia }\end{array}$ & Resoluciones estratégicas \\
\hline $\begin{array}{l}\text { Existencia de objetos } \\
\text { referentes a defender. }\end{array}$ & $\begin{array}{l}\text { ¿Qué objeto/s } \\
\text { referente/s se busca } \\
\text { defender? }\end{array}$ & $\begin{array}{l}\text { Definición del/de los } \\
\text { objeto/s referente/s a } \\
\text { defender. }\end{array}$ \\
\hline $\begin{array}{l}\text { Necesidad de } \\
\text { defenderlos de una } \\
\text { determinada manera. }\end{array}$ & $\begin{array}{l}\text { ¿Cómo se piensa } \\
\text { defenderlo/s? }\end{array}$ & $\begin{array}{l}\text { Establecimiento de las } \\
\text { mejores formas de defender } \\
\text { el/los objeto/s referente/s. }\end{array}$ \\
\hline $\begin{array}{l}\text { Presencia de actores } \\
\text { amenazantes. }\end{array}$ & $\begin{array}{l}\text { ¿De quién/es hay que } \\
\text { defenderlo/s? }\end{array}$ & $\begin{array}{l}\text { Identificación de los actores } \\
\text { que son potenciales } \\
\text { amenazas para el objeto } \\
\text { referente. }\end{array}$ \\
\hline $\begin{array}{l}\text { Presencia de un } \\
\text { escenario en el cual } \\
\text { tienen lugar los riesgos y } \\
\text { amenazas al objeto } \\
\text { referente. }\end{array}$ & $\begin{array}{l}\text { Reconocimiento del } \\
\text { escenario bajo el cual se } \\
\text { deberá defender el objeto } \\
\text { referente. }\end{array}$ \\
\hline
\end{tabular}

Fuente: elaboración propia.

\section{La Dimensión Estratégica de la Política de Defensa desde el caso argentino}

A partir del puente teórico realizado entre los conceptos, la DEPD puede ser definida como la vertiente de la política de defensa que está vinculada a las resoluciones estratégicas de carácter político, en donde los dirigentes de un Estado determinan como objetivo principal la preservación de ciertos activos nacionales y establecen la forma más óptima de hacerlo en consideración de las amenazas externas percibidas y el escenario regional e internacional identificado. Dos cuestiones principales podemos reconocer de la definición. En primer lugar, que la DEPD es de carácter político. Es decir, las definiciones que dicha dimensión propone sobre qué debe defenderse, cómo, de quién y en qué escenario son establecidas por dirigentes políticos y no por militares. En tal sentido, la magnitud y la atención que se le preste a la misma va a estar sujeta a los fundamentos que tengan los políticos (Pion Berlin y Trinkunas, 2007; Battaglino, 2015). En segundo lugar, que si bien la misma parte del plano doméstico al considerar en un primer momento los activos estratégicos a defender, la consideración de las amenazas externas percibidas y del escenario internacional identificado evidencia una conexión entre el nivel nacional y el nivel internacional. 
El caso argentino es de utilidad para ilustrar empíricamente los componentes de la DEPD en la medida que el Ministerio de Defensa del país promueve la elaboración y difusión de documentos oficiales en donde es posible identificar los componentes establecidos por esta vertiente estratégica de la política de defensa. A su vez, las leyes que rigen la defensa nacional argentina, las Directivas Políticas de Defensa Nacional (DPDN) y la predisposición de las autoridades del Ministerio de Defensa a ser entrevistadas también son un insumo importante para analizar esta dimensión.

\section{Primera resolución estratégica: definición de los activos estratégicos a defender}

La aproximación conceptual a la primera resolución estratégica de la DEPD se realizará a partir de la consideración de tres factores. En primer lugar, partimos de la base de que los Estados siempre tienen activos estratégicos objetivos para defender, ya sean recursos naturales, ciudades, instituciones, etcétera. $\mathrm{Al}$ ser esto un hecho invariable, lo relevante para este componente es el nivel de atención que los dirigentes políticos le prestan a la defensa de estos activos. En tal sentido, el déficit de atención puede llegar a niveles tan bajos en donde la dirigencia política ni siquiera identifique los activos estratégicos del Estado. A su vez, el nivel de atención también define qué objetos referentes se van a priorizar, algo que varía en función del escenario internacional identificado y de las amenazas externas percibidas. Esto muestra el vínculo entre el nivel nacional y el internacional, ya que este último tiene impacto en la definición política vinculada a qué activos estratégicos son prioridad para la defensa nacional. En segundo lugar, consideramos que los objetos referentes a defender por el Estado son la base de la DEPD en la medida que su protección es el objetivo político máximo de la misma. Es decir, los otros tres componentes de la DEPD solamente adquieren sentido a partir de los activos estratégicos que deben ser preservados. En tercer lugar, sostenemos que el objeto referente a defender no es solamente la supervivencia del Estado (Waltz, 2010; Mearsheimer, 2014). Si bien la preservación del Estado es el objetivo máximo de cualquier DEPD, este primer componente es más amplio al referir no solo a la supervivencia, sino también a los activos estratégicos que son esenciales para la integridad territorial del Estado y la reproducción y bienestar material de una comunidad. De esta forma, los objetos referentes pueden ser fuentes de recursos natura- 
les, zonas urbanas, puntos de contacto con el exterior, la conectividad interna del Estado y su infraestructura crítica ${ }^{3}$.

En el caso argentino, la ley 23.554 de Defensa Nacional de 1988 define a la misma como "la integración y la acción coordinada de todas las fuerzas de la Nación para la solución de aquellos conflictos que requieran el empleo de las Fuerzas Armadas, en forma disuasiva o efectiva, para enfrentar las agresiones de origen externo" y sostiene, a su vez, que "tiene por finalidad garantizar de modo permanente la soberanía e independencia de la Nación Argentina, su integridad territorial y capacidad de autodeterminación; proteger la vida y la libertad de sus habitantes" (Ley 23.554, art 2). Es destacable el hecho de que el país no define al uso de la defensa nacional únicamente para garantizar la supervivencia del Estado, sino que incluye en la definición a su utilidad para garantizar la integridad territorial. A partir de esto, puede inferirse que el instrumento militar del país es vital para defender activos estratégicos que, aún sin poner en riesgo la autodeterminación del pueblo, su usurpación afectaría negativamente a los intereses del Estado y su ciudadanía. En esta línea, en la entrevista realizada, el primer informante clave indica que si bien durante el CPDN no se realiza un inventario de los objetos referentes que deben ser protegidos por la política de defensa (definición que, en todo caso, debería ser producto de una discusión amplia entre varias áreas de gobierno), la proyección de escenarios futuros relativos al planeamiento por capacidades y vinculado a las hipótesis de empleo del instrumento militar se realiza teniendo en cuenta aquellos activos que están vinculados a los intereses vitales de la nación y que deben ser defendidos. Asimismo, menciona que esta identificación se realiza de forma general en la DPDN y de una manera más concreta y operativa en las etapas siguientes del CPDN y en los informes del Consejo de Defensa Nacional (CODENA), cuyos documentos son clasificados. Un ejemplo establecido para ilustrar lo dicho fue la cuestión relativa a la proyección del instrumento militar hacia el Atlántico Sur y la Patagonia, algo que también se ve reflejado en los documentos oficiales no clasificados. Esta visión es compartida por el segundo informante clave al establecer que, más allá de que la DPDN no identifica en términos concretos qué es lo que hay que defender, la misma deriva en documentos secretos en donde se traducen en el plano operativo aquellas

Consideramos a la 'infraestructura crítica' como "el elemento, sistema o parte de este situado en los Estados miembros que es esencial para el mantenimiento de funciones sociales vitales, la salud, la integridad física, la seguridad, y el bienestar social y económico de la población, cuya perturbación o destrucción afectaría gravemente a un Estado miembro al no poder mantener esas funciones" (Directiva europea 2008/114/CE del 8 de diciembre de 2008). 
cuestiones generales que se mencionan en la DPDN. En este mismo sentido y ligado a los activos estratégicos, el primer informante clave destacó como decisión política de carácter estratégico a la creación del Comando Conjunto de Ciberdefensa en 2014 (a partir de la Resolución 343 del Ministerio de Defensa), ya que el mismo tiene como objetivo proteger la infraestructura crítica del sistema de defensa nacional.

\section{Segunda resolución estratégica: establecimiento de las mejores formas de defender los activos estratégicos}

En lo que respecta a la segunda resolución estratégica, relativa al establecimiento de las mejores formas de defender los objetos referentes, la misma refiere a cómo diseñar el instrumento militar en función de los activos que el Estado busca preservar, por lo que está fuertemente emparentado con el planeamiento estratégico (Anzelini y Poczynok, 2014). Si bien las cuestiones vinculadas al diseño del instrumento militar pueden abordarse de múltiples formas, optamos por tomar las cuatro esferas del instrumento militar reconocidas por Mearsheimer (2014), considerando que cada Estado puede optar por favorecer el diseño y el despliegue del mismo en función de la ubicación de sus activos estratégicos. Las esferas son las siguientes: en primer lugar, la que prioriza el despliegue territorial (Mearsheimer, 2014: 83-137); en segundo lugar, la que hace hincapié en la disposición naval de sus fuerzas (Till, 2007); en tercer lugar, la que pone el énfasis en el poder aéreo; y, en cuarto lugar, la que resalta la importancia de la disuasión nuclear (Lieber, 2000). A su vez, en la actualidad las cuatro están atravesadas por el desarrollo de capacidades estatales orientadas a la inteligencia estratégica militar (Clark, 2007), la actividad espacial y, por último, la protección de la infraestructura crítica de potenciales ciberataques (Nye, 2017; Geis y Hailes, 2016), en donde es fundamental la capacidad de atribución, debido a que en ella radica la posibilidad de identificar qué actores son los ejecutores de éstos (Rid y Buchanan, 2014). Asimismo, la cuestión vinculada al diseño del instrumento militar también tiene en consideración a la forma en que se prevé que actúen las fuerzas que lo componen. En este sentido, su accionar puede ser autónomo, conjunto y, dentro de esta última, puede ser por asociación, contribución o refuerzo (Milia, 1965). Por último, la resolución vinculada a la mejor forma de defender los activos estratégicos identificados incluye no solo a la composición, magnitud, organización y equipamiento del instrumento militar, sino también a su despliegue, doctrina, adiestramiento, comunicaciones y logística. 
Si consideramos el caso argentino a la luz del despliegue de su instrumento militar y de la adquisición de capacidades materiales para defender sus activos estratégicos, podemos ver un desajuste entre las dos primeras resoluciones estratégicas de la DEPD. A pesar de la importancia del Atlántico Sur para el país (Koutoudjian, 2011; Caplan y Eissa, 2015; Magnani, 2020), desde la vuelta a la democracia hasta la actualidad la Argentina no logró ni acomodar el despliegue de su instrumento militar ni priorizar su capacidad naval y terrestre en sus adquisiciones de medios materiales; cuestiones que le permitirían acercarla a su objetivo político de preservar sus activos estratégicos en el Atlántico Sur (Magnani, 2019: 160-170).

\section{Tercera resolución estratégica: \\ identificación de los actores que son potenciales amenazas para el objeto referente}

En un contexto normativo en donde el planeamiento estratégico se realiza por capacidades en lugar de por hipótesis de conflicto (Decreto 1729/ 2007; Canto, Eissa y Gastaldi, 2015), la tercera resolución estratégica, vinculada a la identificación de los actores externos que suponen amenazas para los objetos referentes del Estado, se debe realizar a partir de la proyección de aquellos escenarios que pueden involucrar un daño para los activos estratégicos que el Estado desea preservar. Esta proyección difiere de la cuarta resolución estratégica -reconocimiento del escenario bajo el cual será posible defender el objeto referente- en la medida que consiste en la identificación y análisis de situaciones específicas en donde pueden ponerse en riesgo los objetos referentes particulares, mientras que el cuarto componente de la DEPD refiere al análisis de un escenario internacional más general.

Si bien la proyección de escenarios en función de los activos estratégicos a preservar varía en todos los casos, sostenemos que en la DEPD la identificación de amenazas se realiza a partir del reconocimiento de los actores regionales o extrarregionales que pueden participar en cada escenario específico, su posición relativa de poder en función de la propia (Waltz, 2010) y, lo más importante, a partir del conocimiento intersubjetivo respecto de la identidad y los intereses de los actores que pueden potencialmente participar en dicho escenario (Wendt, 1992). Este último componente nos permite categorizar a los posibles actores como amigos, rivales o enemigos. De esta manera, si bien para realizar estas proyecciones -caracterizadas por el empleo del instrumento militar en forma disuasiva o efectiva- solo es suficiente con que haya un solo actor que sea percibido como rival o enemigo, 
esto no quiere decir que todos los actores del potencial contexto identificado sean un factor de hostilidad.

El ejemplo argentino nos permite ver cómo, a partir de la identificación y evaluación de un escenario particular, pueden reconocerse actores percibidos como potenciales amenazas para la preservación de los objetos referentes. En tal sentido, el primer informante clave menciona que el planeamiento por capacidades del CPDN se realiza en función del juicio por escenarios en donde las amenazas, si bien deben ser estatales y externas, son genéricas y no están claramente identificadas. Asimismo, indica que en función de dichos escenarios se piensa la organización y el despliegue del instrumento militar, en donde desde la gestión se reconoce al Atlántico Sur y a la proyección sobre la Antártida como desafíos que, a pesar de no ser los únicos del sistema de defensa nacional, son esenciales y prioritarios. En esta misma línea, el informante clave también hizo referencia a la capacidad estatal relativa a la vigilancia, el control y el reconocimiento del Atlántico Sur para poder adquirir información sobre los comportamientos de actores extrarregionales en esta demarcación geográfica de interés para el Estado. Evidencia de esto se encuentra en la DPDN (Decreto 2645/2014), en donde no se identifica a Gran Bretaña como un actor amenazante a partir del cual se debe diseñar el instrumento militar, sino que se hace hincapié en definir al Atlántico Sur como un escenario en donde este Estado extrarregional es un actor que, por su capacidad material, acciones e historia conflictiva con la Argentina en lo que respecta a la soberanía de las islas, es visto con preocupación y cautela. En la misma se establece que "la presencia militar británica en el Atlántico Sur genera una tensión innecesaria e injustificada en toda la región” y que el país manifiesta la "preocupación por el despliegue militar británico, que incluye el desplazamiento al Atlántico Sur de submarinos nucleares con capacidad de portar armamento nuclear" (Decreto 2645/ 2014: 6).

\section{Cuarta resolución estratégica: reconocimiento del escenario (internacional y regional) bajo el cual se deberá defender el objeto referente}

La cuarta resolución estratégica hace referencia tanto a la distribución de poder en el escenario internacional como en el regional, estableciendo que las dinámicas de seguridad de ambos niveles afectan a la defensa de los activos estratégicos identificados por los Estados. En este sentido, no solo debemos analizar la distribución de poder en el plano internacional sin un 
anclaje territorial (Waltz, 2010), sino que debemos pensar a la misma en términos geográficos con el objetivo de poder analizar e identificar la capacidad que tienen distintos Estados para participar en los escenarios proyectados por otras naciones y vinculados a la defensa de un activo estratégico. En otras palabras, como la defensa nacional es una acción que busca defender a un objeto localizado dentro del territorio, tanto el poder de los actores internacionales como el de los regionales debe ser medido en función de su capacidad de amenazar con su instrumento militar a los objetos referentes geográficamente situados del Estado cuya DEPD estamos analizando. Esto es fundamental, ya que la presencia de factores naturales que dificultan el desplazamiento del instrumento militar hace que muy pocos actores extrarregionales sean capaces de poner en riesgo los activos de otro Estado (Mearsheimer, 2014: 114-119).

La Teoría de los Complejos Regionales de Seguridad de Buzan y Waever (2003) es de gran utilidad para los propósitos analíticos y conceptuales de este cuarto componente de la DEPD, ya que en la misma se identifican a los actores más importantes del sistema internacional en función de su capacidad de afectar a las dinámicas de seguridad de otras regiones. En este marco, establecen dos tipos de Estados que pueden tener impacto en los asuntos de defensa que exceden su región y, por lo tanto, definen la polaridad del sistema internacional ${ }^{4}$ : los superpoderes y los grandes poderes (Buzan y Waever, 2003: 34-35). A partir del reconocimiento de estos dos tipos de actores, establecemos que los superpoderes son aquellos Estados que tienen un instrumento militar y la capacidad económica para desplegar su aparato de defensa en todas las regiones del mundo; mientras que los grandes poderes tienen la capacidad para afectar las dinámicas de seguridad y los cálculos de seguridad de Estados de otras regiones cercanas a las suyas, aunque no de todo el mundo.

En función del uso de esta distinción entre el nivel regional y el internacional, en la parte correspondiente al segundo, los Estados (1) reconocen la polaridad del sistema internacional, (2) identifican los actores estatales de dicho sistema que tienen la capacidad material de afectar sus activos estratégicos y (3) establecen qué tipos de intereses tienen estos actores y cuáles son los tipos de relaciones que tienen entre ellos $^{5}$ con el objetivo de poder dar cuenta de los niveles de pugnacidad del escenario internacional. Por

\footnotetext{
4 Entendemos por el término "polaridad" a la distribución de poder en un determinado sistema, ya sea este regional o internacional.

5 En este punto es conveniente hacer referencia a los tres tipos de culturas -hobbesiana, lockeana o kantiana- que pueden tener los sistemas internacionales que establece Wendt (1992).
} 
otro lado, con respecto a la parte correspondiente al nivel regional de la cuarta resolución estratégica -y utilizando también como base teórica los lineamientos de los complejos de seguridad regional de Buzan y Waever $(2003)^{6}-$, los Estados buscan reconocer de su escenario regional (1) la distribución relativa de poder, (2) las intenciones del resto de los actores estatales de la región y (3) el tipo relaciones interestatales que mantienen estos actores $\left(\mathrm{amistad} / \mathrm{rivalidad} / \mathrm{enemistad}^{7}\right)$. A partir de una variación en estos elementos identificados tanto del escenario internacional como del regional, los Estados pueden modificar las percepciones sobre cuáles son potenciales amenazas y esto, en consecuencia, puede incidir en la prioridad de los activos estratégicos a defender y reformular el diseño y despliegue de su instrumento militar. En este sentido, la cuarta resolución estratégica de la DEPD tiene un impacto en las primeras tres de forma secuencial, mostrando cómo un cambio en la apreciación del escenario puede impactar en todos los otros componentes de la misma.

En función del caso argentino, el análisis que hace el país de su escenario global es de marcada relevancia a la hora de realizar el CPDN. La autoridad consultada del Ministerio de Defensa establece que en cada escenario identificado incluyen actores más poderosos pugnando, en donde se reconocen a Estados Unidos, China y en menor medida Rusia. A su vez, marca que estas disputas de los actores más poderosos del sistema internacional requieren decisiones complejas tanto de defensa nacional como de política exterior, en las que la utilización del instrumento militar se vuelve solo una opción de ultima ratio para defender los intereses nacionales en cada escenario identificado. Este análisis de los niveles de pugnacidad entre potencias se plasma en la DPDN, al identificar que "se ha producido un fenomenal proceso de redistribución del poder global como consecuencia del fin de la Guerra Fría en 1991", en donde "se pasó entonces de una estructura bipolar de distribución del poder a una multipolar, con los ESTADOS UNIDOS DE AMÉRICA como única superpotencia remanente” (p. 2). Si bien en cuanto a capacidades en el documento se identifica que "ESTADOS UNIDOS DE AMÉRICA, considerándolo individualmente, detenta sobre el resto de los Estados del sistema internacional una supremacía indiscutible (...)

\footnotetext{
6 Específicamente, retomamos de la construcción conceptual de Buzan y Waever el hecho de que los complejos regionales de seguridad tienen entre sus componentes a la distribución regional de poder y a los patrones de amistad y enemistad entre los Estados que componen la región.

$7 \quad$ Esta caracterización hace referencia a los distintos tipos de relaciones que tienen los Estados en función de sus patrones intersubjetivos de relacionamiento (Ver Wendt, 1992).
} 
y una efectiva capacidad para proyectar globalmente recursos de poder" (p.3), la autoridad del Ministerio de Defensa consultada establece que actualmente China y en menor grado Rusia se presentan como actores que, por su capacidad y el grado de disputa que mantienen con Estados Unidos de América, hay que tener en consideración en el juicio por escenarios. Esto está bien ilustrado en la misma DPDN, en donde si bien se marca la supremacía de Estados Unidos, se reconocen progresivos "cambios en la distribución de poder global, tanto militar como económico” (p.3). Asimismo, la directiva destaca el surgimiento de un nuevo paradigma de la defensa nacional, vinculado con las tecnologías de la información, en donde "la dimensión ciberespacial, sin locación física específica propia, genera replanteos sobre las tradicionales categorías con las que se aborda la "guerra real" y exige, por la dinámica propia de la innovación tecnológica, una rápida adaptación para los Sistemas de Defensa" (p.4).

Con respecto al escenario regional, si bien la directiva no identifica la distribución de poder regional, reconoce en la región sudamericana "un encadenamiento positivo entre los procesos anteriormente mencionados y la progresiva disminución de las percepciones de amenaza y las competencias estratégicas y militares", en donde "esta nueva dinámica abrió paso al inicio de procesos de establecimiento de medidas de confianza mutua e interacción y cooperación en materia de Defensa” (p.5). En la entrevista realizada, el segundo informante clave indica que estos procesos de interacción regional contribuyeron al reconocimiento y a la definición política de que Argentina necesitaba aliarse con sus pares regionales para poder defender sus intereses de potenciales escenarios adversos que incluyan actores extrarregionales.

Tres cosas pueden ilustrarse a partir del caso argentino con respecto al potencial impacto del reconocimiento del escenario internacional y regional en los otros componentes de la DEPD. Por un lado, que la ausencia de un escenario regional pugnaz que permita percibir como amenazas a otros Estados de la región probablemente llevó a los dirigentes políticos argentinos en sus documentos oficiales a no proyectar escenarios vinculados a la preservación de activos estratégicos localizados cerca de zonas de frontera como una prioridad para la defensa nacional. Por otro lado, el reconocimiento de un superpoder con capacidad de desplegar su instrumento militar en todo el mundo junto con la identificación de un escenario regional apacible pudo haber incidido en la voluntad política de los formuladores de políticas del Estado argentino de incluir dentro de las misiones subsidiarias de su instrumento militar a la participación de éste en la construcción de un sistema de defensa subregional que excluya a este superpoder (Decreto 1691/2006). En 
este sentido, es posible considerar que la cuarta resolución estratégica de la DEPD tiene la capacidad de incidir en la formación de alianzas de un Estado a partir de la identificación de qué actores del escenario internacional son compatibles con la protección de los activos estratégicos que el Estado busca preservar. Por último, el reconocimiento de nuevas tendencias de seguridad y militares en el escenario internacional, como lo es el ciberespacio, puede tener implicancias en los objetos referentes a defender priorizados por el Estado, hecho que se encuentra ejemplificado en el caso argentino a partir de la decisión política de crear el Comando Conjunto de Ciberdefensa en 2014.

\section{La Dimensión Estratégica de Defensa y la relación entre sus componentes}

A lo largo del artículo establecimos que, dentro del espectro de los fundamentos políticos, la DEPD se desarrolla a partir de las cuatro resoluciones estratégicas identificadas, en donde la primera, relativa a qué activos estratégicos a preservar son priorizados por el Estado, es el componente más importante de la misma en la medida que los otros tres se desarrollan a partir de éste. En tal sentido, consideramos que los Estados tienen activos estratégicos fijos a defender -recursos naturales, zonas urbanas, puntos de contacto con el exterior, conectividad interna e infraestructura crítica- que no pueden ser cambiados, ya que afectan de manera objetiva a la seguridad y el bienestar de la comunidad. No obstante, en términos metodológicos, esta primera resolución de la DEPD puede considerarse como variable, ya que lo que cambia de la misma son las prioridades políticas respecto a qué activos estratégicos se deben defender. Es decir, del stock de activos estratégicos objetivos que los Estados tienen para defender -algo que varía según el Estado-, los políticos toman definiciones con respecto a cuáles son prioritarios en función de las otras resoluciones estratégicas.

La cuestión clave que tienen en consideración los decisores políticos para definir cuáles son los objetos referentes que tienen prioridad es la vinculada a la cuarta resolución estratégica, en donde se reconocen los planos internacional y regional midiendo los niveles de pugnacidad de ambos escenarios a partir de la identificación de cuáles son las intenciones y los intereses de los actores más importantes en términos de distribución de poder. Esto les permite dar cuenta de los escenarios que pueden comprometer la preservación de los activos estratégicos del Estado y, a partir de los mismos, percibir a aquellos actores estatales que son potencialmente hostiles en dicho escenario (tercera resolución estratégica). A partir de esto, se logran 
identificar aquellos activos estratégicos que tienen mayores probabilidades de correr riesgo (primera resolución estratégica) y se diseña el instrumento militar (segunda resolución estratégica) para proteger dichos activos. Esta secuencia muestra que la DEPD funciona como una correa de transmisión, que comienza en el reconocimiento de los escenarios internacionales y regionales, pasa a definir a aquellos actores hostiles a partir de los cuales se establecen los objetos referentes que se deben defender con prioridad $y$, por último, culmina con la definición de un determinado diseño del instrumento militar.

Ahora bien, es importante realizar dos precisiones. En primer término, hay que remarcar que, si bien establecemos que los Estados tienen activos estratégicos para defender que son objetivos, ergo permanentes, la prioridad de los mismos es una definición enteramente política que depende de factores que escapan a la objetividad y permanencia de los objetos referentes. En este sentido, puede haber objetos referentes que ni siquiera sean identificados por la dirigencia política como activos a defender, a pesar de que los mismos sean de una importancia sustantiva para la supervivencia y el bienestar de la comunidad. En un segundo orden, la DEPD evidencia la fuerte conexión que existe entre la política de defensa de un Estado y el escenario internacional y regional en el cual este se encuentra posicionado. De tal forma, si bien la vertiente estratégica de la defensa nacional parte de las certezas nacionales -activos estratégicos a defender-, la misma siempre se define a partir de una reflexión situada respecto al escenario externo. Metodológicamente, podemos establecer que dentro de la DEPD las resoluciones estratégicas tres y cuatro son las variables independientes mientras que las resoluciones uno y dos son las dependientes.

\section{Reflexiones finales}

El presente artículo ha tenido como objetivo contribuir al campo de los estudios sobre defensa nacional al llamar la atención sobre una cuestión parcialmente desatendida por la literatura. En este sentido, se introdujo a la Dimensión Estratégica de la Política de Defensa como un término que busca ampliar el abordaje teórico y conceptual de estos estudios. El caso argentino fue utilizado para ilustrar empíricamente la construcción teórica de la DEPD a partir de los documentos oficiales correspondientes a la defensa nacional de un país, dejando para futuros trabajos la posibilidad de realizar un estudio de caso para establecer la correlación causal respecto de cómo la variación de uno de los componentes de la DEPD tiene impacto en el resto. 
Asimismo, la introducción de la DEPD tiene la potencialidad de abrir nuevas líneas de investigación sobre la defensa nacional, al establecer una base teórica para indagar con respecto a cómo varían las prioridades de defensa de los Estados junto con el diseño y el despliegue de su instrumento militar en función de los cambios en los entornos estratégicos regional e internacional. A su vez, el concepto introducido hace posible ampliar el espectro de análisis respecto del posible impacto de los organismos de seguridad regional e internacional en las políticas de defensa de los Estados, permitiendo no solo centrar el análisis en cómo dichas organizaciones afectan al gobierno político de la defensa o a la cooperación en materia de seguridad, sino también analizar su impacto en la vertiente estratégica de la política de defensa de los Estados. Asimismo, la DEPD brinda la oportunidad de revitalizar los estudios vinculados a las relaciones cívico-militares, abriendo la posibilidad de que se investigue con respecto a cuál es el rol de la dirigencia política en la definición de las cuatro resoluciones estratégicas y, de esta forma, analizar desde otra perspectiva los niveles de subordinación militar a la política.

Para finalizar, es relevante destacar que, considerando que los Estados tienen activos estratégicos permanentes a defender y que constituyen la base de la dimensión estratégica de la política de defensa, es tarea de los investigadores indagar respecto de cuáles son las prioridades políticas, por qué y qué factores condicionan la jerarquización de éstas. Solo de esta manera podremos tener un análisis más completo de la política de defensa y de su estrecha vinculación con el plano internacional.

\section{Referencias bibliográficas}

Anzelini, L. y Poczynok, I. (2014). El Planeamiento Estratégico Militar en la Argentina (2003-2013): Reflexiones en torno al gobierno político de la defensa. Revista Brasileira de Estratégia Ẽ Relaçoes Internacionais, 3(6), 143-167.

Anzelini, L. Poczynok, I. y Zacarías, M. E. (2017). Política de defensa y militar en Argentina desde el retorno a la democracia (1983-2015). Serie Documentos de Trabajo. Universidad Metropolitana para la Educación y el Trabajo, 4, 1-68.

Battaglino, J. (2010). "La política militar de Alfonsín: la implementación del control civil en un contexto desfavorable". En R. Gargarella; M. Murillo; M. Pecheny (Eds.), Discutir Alfonsín (Pp. 161-184). Buenos Aires: Siglo XXI.

Battaglino, J. (2012). Política de defensa y política militar durante el kirchnerismo. En A. Malamud y M. De Luca (Eds.), La política en tiempos de los Kirchner (Pp. 241-250). Buenos Aires: Eudeba. 
Battaglino, J. (2015). Fundamentos Olvidados de la Política de Defensa: Reflexiones a partir del Caso Argentino. Revista Brasileria de Estudos de Defesa, 2(2), 197-2016.

Buzan, B. y Waever, O. (2003). Regions and Powers. Cambridge: University Cambridge Press.

Bueno, A. (2018). De los Estudios Estratégicos. Conceptualización y evolución de un campo de estudio. Revista de Estudios en Seguridad Internacional, 4(1), 237-256.

Canto, M. B., Eissa, S. y Gastaldi, S. (2015). Postura estratégica y planeamiento para la defensa en la República Argentina. Un aporte para el debate. Serie documentos de trabajo, Escuela de Defensa Nacional, 31, 4-17.

Caplan, S. y Eissa, S. (2015). Análisis estratégico del Sistema Malvinas, Antártida y Atlántico Sur. En Documentos de Trabajo $N^{o} 28$, Buenos Aires, Escuela de Defensa Nacional.

Clausewitz, K. (2015). De la guerra. Buenos Aires: Ensayos agebe.

Clark, R. J. (2007). Intelligence and National Security. Westport : Praeger security international.

Derghougassian, K. (2012). Evolución de la política de defensa. En La defensa en el Siglo XXI. Argentina y la seguridad regional. Buenos Aires: Capital Intelectual.

Eissa, S., Canto, M., Gastaldi, S., y Justribó, C. (2013). El modelo de defensa no provocativa como postura estratégico-militar para la Argentina. Revista Espacios Políticos, 9.

Eissa, S. (2013a). Redefiniendo la política de defensa: hacia un posicionamiento estratégico defensivo regional. Revista SAAP, 7(1), 41-64.

Eissa, S. (2013b). Política exterior y política de defensa en Argentina: dos caras de la misma moneda. Perspectivas de Políticas Públicas, 3(5), 171-191.

Garnett, J. (1987). Strategic Studies and Its Assumptions. En Baylis, J., et. al. Contemporary Strategy. Nueva Jersey: Holmes \& Meier Publishers.

Geis, P. y Hailes, J. T. (2016). Deterring Emergent Technologies. Strategic Studies Quarterly, Fall, 47-73.

Gray, C. S. (1999). Clausewitz rules, OK? The future is the past-with GPS. Review of International Studies, 25(5), 161-182.

Gray, C. S. (2015), The Future of Strategy. Cambridge: Polity Press.

Heuser, B. (2010). The Evolution of Strategy: Thinking War from Antiquity to the Present. Cambridge,: Cambridge University Press.

Kane, T. y Lonsdale, D. J. (2012). Understanding Contemporary Strategy. Londres: Routledge.

Koutoudjian, A. (2011). Lineamientos para la incorporación de la problemática del mar argentino en la planificación territorial. Buenos Aires: Subsecretaria de planificación territorial de la inversión pública.

Liddell Hart, B. H. (1967). Strategy. Londres: Faber \& Faber.

Lieber, K. A. (2000). Grasping the Technological Peace. The Offense-Defense Balance and International Security. International Security, 25(1), 71-104. 
López, E. (1988). El último levantamiento. Buenos Aires: Legasa.

Magnani, E. (2019). Los Estados Periféricos frente a sus intereses geopolíticos: el caso de la Argentina con el Atlántico Sur. Revista POSTData, 24(1), 139-176.

Magnani, E. (2020), La dimensión geopolítica del interés estatal: el Atlántico Sur Occidental y su relevancia para la Argentina. Revista Relaciones Internacionales, 93(1), 13-33.

Mearsheimer, J. J. (2014). The Tragedy of Great Power Politics. Nueva York:: W. W Norton \& Company.

Milia, F. A. (1965). Estrategia y Poder Militar. Buenos Aires: Instituto de Publicaciones Navales.

Nye, J. (2017). Deterrence and Dissuasion in Cyberspace. International Security, 41(3), 44-71.

Pion-Berlin, D. y Trinkunas, H. (2007). Attention Deficits: Why politicians Ignore Defense Policy in Latin America. Latin American Research Review, 42(3), 76-100.

Pion-Berlin, D. y Martinez, R. (2019). Soldiers, Politicians, and Civilians: Reforming Civil-Military Relations in Democratic Latin America. Cambridge: Cambridge University Press.

Ramos, H. (2015). Los estudios sobre defensa en Argentina (1983-2014). Defensa Nacional y Pensamiento Estratégico, 2, 5-50.

Rid, T. y Buchanan, B. (2015). Attributing Cyber Attacks. Journal of Strategic Studies, 38(1), 4-37.

Saín, M. (2003). "Nuevos horizontes, nuevos problemas. Las Fuerzas Armadas argentinas frente a las "Nuevas Amenazas" (1990-2001)”. En E. Lópezy M. Saín. (Eds.). Nuevas Amenazas. Dimensiones y perspectivas. Dilemas y desafios para la Argentina y Brasil (Pp. 173-220). Buenos Aires: Universidad Nacional de Quilmes.

Saín, M. (2010). Los votos y las botas. Estudios sobre la defensa nacional y las relacionesciviles militares en la democracia argentina. Buenos Aires: Prometeo Libros.

Scheetz, Ty Cáceres, G. (1995). Defensa no provocativa. Una propuesta de reforma militar para la Argentina. Buenos Aires: Buenos Aires Editora.

Till, G. (2007). Poder Maritimo, una guia para el siglo XXI. Buenos Aires: Instituto de Publicaciones Navales.

Vennesson, Paul (2017). Is strategic studies narrow? Critical Security and the Misunderstood Scope of Strategy. Journal of Strategic Studies. 40(3), 358-391.

Waltz, K. (2010). Theory of International Politics. Illinois: Waveland Press, Inc.

Wendt, A. (1992). Anarchy is what States Make of it: The Social Construction of Power Politics. International Organization, 46(2), 391-425. 


\section{Documentos oficiales}

Decreto 727/2006

Decreto 1691/2006

Decreto 1729/2007

Decreto 1714/2009

Decreto 571/2020

Directiva europea 2008/114/CE del 8 de diciembre de 2008

Directiva Política de Defensa Nacional. Decreto 2645/2014

Ley 23.554 de Defensa Nacional (1988)

Ley 24.059 de Seguridad Interior (1992)

Ley 25.520 de Inteligencia Nacional (2001)

Libro Blanco de Defensa Argentina (2015)

Resolución 343/2014 del Ministerio de Defensa.

\section{Entrevistas}

Primer informante clave - Participó en el CPDN durante los años 2007-2014. Fecha de realización de la entrevista: 9/02/2021.

Segundo informante clave - Participó en el CPDN durante los años 2015-2019. Fecha de realización de la entrevista: 11/02/2021. 